\title{
Hypertension, Obesity, and COVID-19: a Collision of Pandemics
}

\author{
Annalisa Perez ${ }^{1} \cdot$ Mihran Naljayan $^{1} \cdot$ Imran Shuja $^{1} \cdot$ Andre Florea $^{1} \cdot$ Efrain Reisin ${ }^{1}$ \\ Accepted: 1 June 2021 / Published online: 29 June 2021 \\ (C) The Author(s), under exclusive licence to Springer Science+Business Media, LLC, part of Springer Nature 2021
}

\begin{abstract}
Purpose of Review To highlight the epidemiology and pathophysiology of hypertension and obesity in COVID-19 infection Recent Findings Hypertension and obesity have emerged as significant risk factors for contracting the COVID-19 virus and the subsequent severity of illness. ACE2 receptor expression and dysregulation of the RAAS pathway play important roles in the pathophysiology of these associations, as do the pro-inflammatory state and cytokine dysregulation seen in obesity. Some of these patterns have also been seen historically in other viral illnesses.

Summary Understanding the mechanisms behind the associations between COVID-19, hypertension, and obesity is important in developing effective targeted therapies and monitoring vaccine response and efficacy. More research is needed to apply our growing knowledge of the pathophysiology of COVID-19, hypertension, and obesity to prevention and treatment.

Interventions focusing on lifestyle modification in managing hypertension and obesity can potentially have a positive impact on containing this pandemic and future viral illness outbreaks.
\end{abstract}

Keywords COVID-19 $\cdot$ Hypertension $\cdot$ Obesity $\cdot$ ACE2 receptor $\cdot$ RAAS

\section{Introduction}

In this review, we explore the pathophysiologic links between hypertension, obesity, and COVID-19 infection. Obesity and hypertension have been shown to be associated with viral illnesses, including influenza, HIV, and other coronaviruses [1,2,3••] Understanding the mechanisms behind these associations may help bring this current pandemic under control, and also contain future viral outbreaks before they reach such epic proportions.

\section{COVID-19 and Hypertension}

Hypertension is categorized as stage 1 (SBP $130-139 \mathrm{~mm}-\mathrm{Hg}$ or DBP $80-89 \mathrm{~mm}-\mathrm{Hg}$ ) and stage 2 (SBP $\geq 140 \mathrm{~mm}-\mathrm{Hg}$ or $\mathrm{DBP} \geq 90 \mathrm{~mm}-\mathrm{Hg}$ ). Most of the studies in this section do not specify the stage of hypertension, but do identify patients with a history of hypertension at the time of COVID-19 diagnosis.

Topical Collection on Hypertension and Obesity

Annalisa Perez

apere5@1suhsc.edu

1 Department of Internal Medicine, Section of Nephrology and Hypertension, Louisiana State University, 1542 Tulane Avenue Suite 330, New Orleans, LA 70112, USA
The association between COVID-19 and hypertension was noted in the first studies of the outbreak. Huang et al. [4] published a case series of 41 patients in China who were positive for SARS-CoV-2, of whom $15 \%$ had hypertension. The median reported age was 49 , and $73 \%$ were male. In an early publication on a COVID-19 patient cohort in New York City, the most common comorbidity was hypertension, reported to be present in $50.1 \%$ of the 393 patients [5]. Richardson et al. [6••] found that the presence of hypertension was the most common comorbidity $(56.6 \%)$ in a larger cohort of 5700 COVID-19 patients in New York. In a study of approximately 800 patients in New Orleans, $73.8 \%$ of those hospitalized with COVID-19 had hypertension. Hypertension, obesity, and diabetes, as well as Black race, were the most common risk factors in the hospitalized group [7].

This association between hypertension and COVID-19 is a global phenomenon. Schonfeld et al. [8] reported hypertension in $19.2 \%$ of 207,079 patients diagnosed with COVID-19 in Argentina. They also found that hypertension, older age, and male sex were associated with worse outcomes in this cohort. In a meta-analysis by $\mathrm{Ng}$ et al. [9] of approximately 375,000 patients from 14 countries-Brazil, China, India, Italy, Mexico, Oman, Saudi Arabia, South Korea, Spain, Turkey, Uganda, UK, and the USA - hypertension (21.3\%), obesity $(18.3 \%)$, and diabetes $(18.1 \%)$ were the most prevalent comorbidities. 
Hypertension has also been found in patients with COVID19 requiring ICU admission. In a retrospective, observational cohort study in Italy, Grasselli et al. [10] reported that, among 3988 patients with COVID-19 admitted to the ICU, hypertension was the most common comorbidity, and having hypertension increased mortality risk. This finding was also seen in a study of COVID-19 ICU admissions in a US population [11].

COVID-19 not only affects older adults, but also has spread rapidly among young adults (18-34 years old). In a dataset of 3222 young adults, Cunningham et al. [12] reported that $57 \%$ were Black or Hispanic and $16.1 \%$ had hypertension. In this group, hypertension, obesity, and male sex were associated with greater risk of death or mechanical ventilation, but neither race nor ethnicity increased this risk.

In summary, as evidenced by many published studies, patients with baseline hypertension were more likely to be diagnosed with COVID-19 and to have more severe disease, as indicated by requiring ICU admission and mechanical ventilation and by having a higher mortality $[4,5,6 \bullet \bullet, 7-11]$ Though it may be possible that patients with hypertension were more likely to express COVID-19 symptoms, and therefore more likely to be tested and seek medical treatment, these studies suggest that patients with underlying hypertension were more likely to be infected and have worse outcomes.

\section{Pathophysiology of Hypertension and the RAAS Pathway in COVID-19}

COVID-19 has been shown to cause cardiovascular and respiratory complications as well as hypercoagulability in its hosts $[13 \cdot \bullet]$. How this occurs may be related in part to the relationship between the COVID-19 virus and the reninangiotensin-aldosterone system (RAAS) (see Fig. 1).

The RAAS pathway is complex and involves a cascade of hormones and various receptors that interplay with each other, enabling blood pressure control and maintenance of fluids and electrolytes in the human body. Many factors are important in the regulation of this system, including decreases in glomerular afferent arteriole pressure and increases or decreases in sodium concentration in the distal tubule of the nephron [13•0].

Activation of the RAAS pathway begins with angiotensinogen from the liver being cleaved to angiotensin I by renin, which is released by the kidney in response to decreased sodium delivery to the juxtaglomerular apparatus. Angiotensin I is then converted to angiotensin II by the angiotensin-converting enzyme (ACE), which is highly expressed in the lung tissue. Angiotensin II activates angiotensin 1 receptors located in the heart, vasculature, kidney, and adrenal cortex, resulting in vasoconstriction and blood pressure elevation. Angiotensin-converting enzyme 2 (ACE2) converts angiotensin I to angiotensin 1-9 and angiotensin II to angiotensin 1-7, which directly act on the Mitochondrial Assembly Receptor 1, leading to vasoprotective antiproliferative and anti-inflammatory effects [13・•].

Earlier studies have shown that ACE2 is the entry point for the novel coronavirus [13••]. The coronavirus inserts itself into the catalytic site of membrane-bound ACE2 for host cell entry [14]. This causes downregulation of ACE2 expression, such that the enzyme can no longer exert its protective effects in various organs. Unchecked angiotensin II activity follows, which has been hypothesized to play a major role in the various organ injuries that occur in COVID-19 [15]. In addition

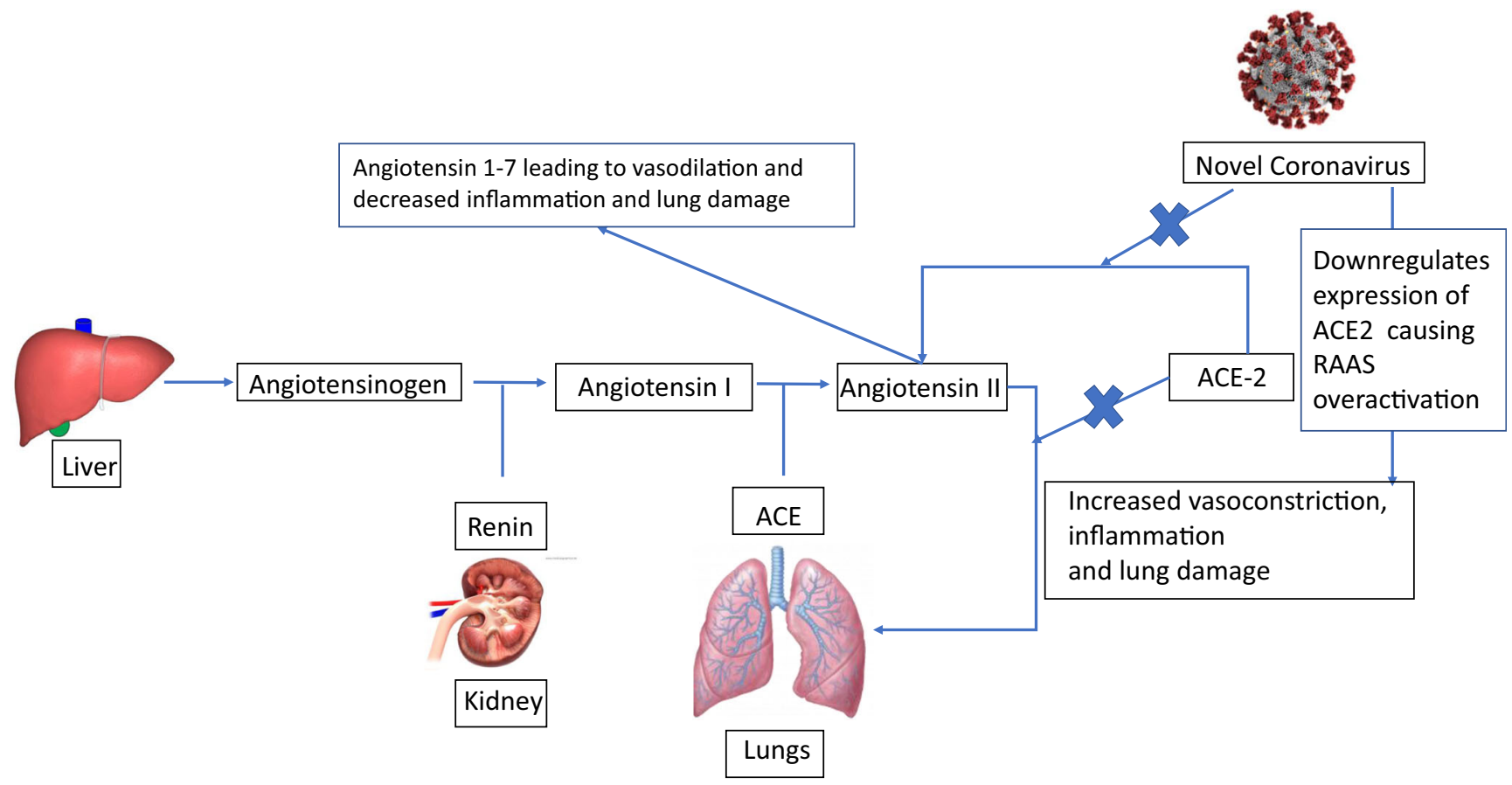

Fig. 1 The relationship between RAAS and COVID-19 
to its vasoconstrictor effects, angiotensin II is associated with endothelial dysfunction and inflammation [13••]. A study by Liu et al. [16] has found that viral load and lung injury that occur in COVID-19 infected patients are associated with increased levels of angiotensin II.

ACE2 has been found in a variety of organs, including the liver, kidney, brain, lymph nodes, lung, and stomach. Thus, ACE2 downregulation may be a possible explanation for the widespread extra-pulmonary effects of COVID-19 in human patients. The ACE2 receptor has been shown to be a target for other coronaviruses as well, such as (SARS)-CoV and CoVNL63. 117

Another recently proposed mechanism for the deleterious effects of COVID-19 in the lung tissue is increased activation of the bradykinin 1 and 2 receptors as a result of downregulation of ACE2. Activation of bradykinin 1 and 2 receptors leads to angioedema and vascular leakage into the lung parenchyma [17].

Other investigators have shown that plasma ACE2 can remain overexpressed in humans months after they were infected by the novel coronavirus [14]. Since the coronavirus enters host cells via the catalytic site of membrane-bound ACE2, Patel et al. [14] hypothesized that infection with COVID-19 will lead to shedding of ACE2 from cell membranes and increased plasma levels. They showed that plasma ACE2 remains elevated even in those with mild or moderate COVID-19. This may explain the deleterious effects of COVID-19 experienced by "long haulers," those patients who have persistent severe symptoms months after the original infection [14].

Given the high prevalence of ACE inhibitor and angiotensin receptor blocker (ARB) use for the treatment of hypertension, studies have examined whether these medications may have negative effects on patients with COVID-19 [18]. Ren et al. [19] explored whether patients taking various antihypertensive medications including ACE inhibitors, ARBs, beta-blockers, calcium channel blockers, and thiazide diuretics were at increased risk of being diagnosed with COVID-19 or having severe COVID-19. They did not find a correlation between the use of any specific anti-hypertensive medication and the increased risk of either of these conditions. Another meta-analysis published in March 2021 also concluded that there was no mortality difference between COVID-19 patients taking ACE inhibitors and those who did not [20].

The latest research on the role of the RAAS pathway in COVID-19 focuses on genetic variations in ACE2 expression among individuals [21]. A publication by Lanjanian et al. [21] found that some missense variants of the ACE2 receptor have reduced affinity for the COVID-19 spike protein that gains entry into cells. Further investigation in this area may help target future therapies for COVID-19.

Wysocki et al. [22•] have fused a soluble ACE2 protein with an albumin-binding domain tag that extends the duration of action of soluble ACE2 from about 8 to $96 \mathrm{~h}$. Using this engineered ACE2 protein as substrate "bait" for the COVID19 virus may have the beneficial effects of both blocking viral entry into cells and limiting their replication, and restoring ACE2 activity and the balance of angiotensin II and bradykinins [22•].

The RAAS is complex, and the particular role that ACE2 plays in propagating COVID-19 infection and in the resulting dysregulation of the RAAS pathway requires further research. More studies on the association between the RAAS, hypertension, and COVID-19 infection may lead to more targeted therapeutic interventions in the future.

\section{COVID-19 and Obesity}

Obesity is defined as excessive fat accumulation that may impair health. The World Health Organization (WHO) uses the following classification system for obesity: Class I is a Body Mass Index (BMI) of 30-34.9; Class II is a BMI of 35-39.9; and Class III is a BMI of $>40$. The WHO estimates that approximately $13 \%$ of the world's adult population is obese [23]. The Centers for Disease Control (CDC) reports an even higher prevalence of obesity in US adults, ranging from 40.3 to $46.4 \%$ in men based on age group and 39.7 to $43.3 \%$ in women [24]. It is estimated that worldwide obesity rates have nearly tripled since 1975 and that more than 650 million adults are obese [24].

Early in the COVID-19 pandemic, obesity emerged as a leading risk factor for both contracting the infection and severity of illness. In a meta-analysis examining the risk factors for COVID-19 infection in a variety of countries, 18 of 20 studies found that obesity was associated with an increased risk of infection. Pooled data analysis showed that the odds of obese individuals being COVID-19 positive were $46 \%$ higher than in those who were not obese. Among COVID-19 patients with symptoms, those who had severe or critical conditions had higher BMIs than those with mild illness and were more likely to require ICU admission. The majority of studies also showed that obesity was associated with an increased risk of in-hospital mortality [25].

In an analysis of 88 US hospitals and a total of 7602 patients, Hendren et al. [26] found that, after multivariate adjustment, increasing class of obesity was associated with a higher risk of in-hospital death or mechanical ventilation. This effect was more pronounced in patients younger than 50 of age. Obesity was also associated with higher risks of dialysis initiation and thromboembolism [26]. Other studies have shown similar increases in risk in the highest class of obesity and in younger obese patients [27].

In the previously mentioned publication by Richardson et al. [6••] of 5700 patients in New York City hospitalized for COVID-19 infection, the second most common comorbidity after hypertension was obesity (41.7\%). The average 
prevalence of obesity in New York City residents overall was $22 \%[6 \cdot \bullet]$. Obesity also appeared to be a risk factor for mechanical ventilation. The rate of mechanical ventilation was 10 times higher than that reported in a study from China [5]. Although the authors did note that this great disparity may be related to different intubation criteria in the two settings, it is notable that, in the study by Cai et al. [28], only $10.7 \%$ of the 383 patients studied were considered obese. They also described an increased risk of more severe infection in obese patients, with an odds ratio of 2.42 of developing severe pneumonia [28].

More than a year into the pandemic in the USA, the association between obesity and COVID-19 has been further strengthened. In a large US study of 148,494 adults testing positive for COVID-19 in 238 hospital emergency rooms across the country between March and December 2020, $50.8 \%$ were obese. Obesity was a risk factor for hospitalization and death, particularly among those over 65 years old, and the risk increased with increasing level of obesity [29•].

These epidemiological data have significant implications for controlling the spread of COVID-19, as well as for the prognosis and management of those affected. Communities where obesity is more prevalent are likely to experience a greater burden of infection and more severe cases and mortality [29•]. Understanding the mechanism underlying obesity as a risk factor can potentially lead to the development of more targeted therapies and help guide vaccine recommendations.

\section{Pathophysiology of Obesity in COVID-19}

The association between obesity, viral pathogenesis, and the course of infection is not unique to COVID-19. Research dating back to the Spanish Influenza pandemic more than a century ago yielded the same findings [3••]. More recently in the H1N1 influenza and $\mathrm{H} 5 \mathrm{~N} 1$ influenza outbreaks, obesity was again linked to increased severity of disease and risk of mortality [3••, 30].

Multiple mechanisms are thought to contribute to the association between obesity and COVID-19 infection (see Fig. 2). The mechanical stress of morbid obesity can increase airway resistance and reduce tidal volume, expiratory reserve volume, and compliance of the lungs [3••]. These changes make the virus's effect on the lungs more dangerous, leading to an increased requirement for intubation and a decrease in the ability of the lungs to recover from intubation. The need for mechanical ventilation due to COVID-19 has been shown to increase mortality, especially when the patient requires it for an extended period $[6 \bullet \bullet]$. The increased work of breathing that is caused by decreasing functional lung capacities in the setting of obesity can exacerbate the acute respiratory distress syndrome (ARDS) in patients with COVID-19 [3••]. Impaired ventilation of the more highly perfused lower lungs in obese patients can result in ventilation-perfusion mismatch and worsening hypoxia $[3 \cdot \bullet]$.

Obesity is often associated with hypertension, type 2 diabetes, metabolic syndrome, and chronic kidney disease

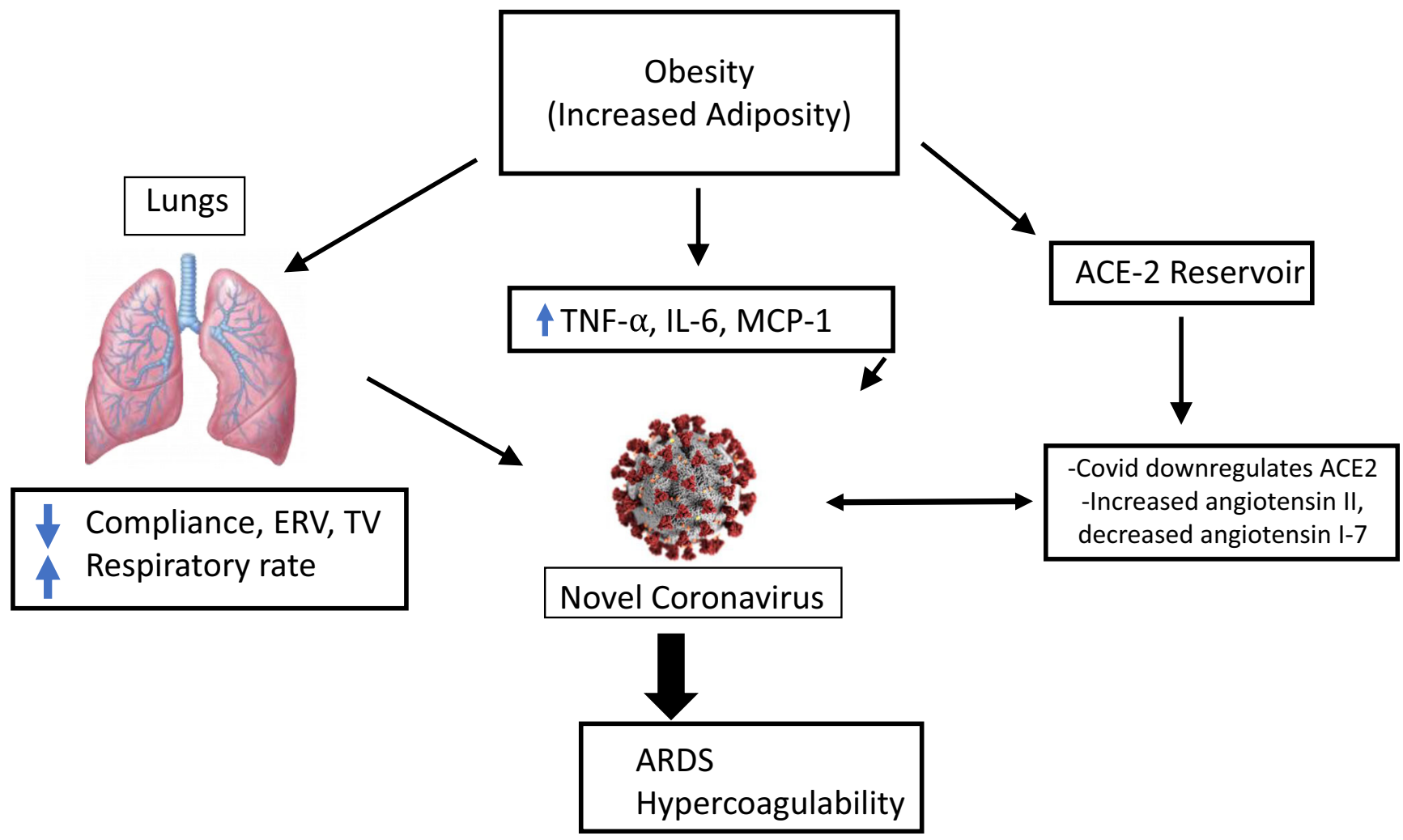

Fig. 2 The role of obesity in COVID-19 
(CKD), all of which have been shown to result in poorer COVID-19 outcomes. Ischemic heart disease, stroke, diabetes, and CKD are among the top ten causes of death in developed countries [31]. Each of these processes can be linked to obesity and to each other with high levels of statistical significance, suggesting that preventing or reversing obesity is one of the most effective means of reducing the morbidity and mortality related to these chronic conditions.

The low-grade systemic inflammation related to obesity can interfere with the immune system's response to viruses, as shown in increased mortality from the influenza virus in obese persons. Obesity is a chronic state of inflammation resulting from overexpression of pro-inflammatory cytokines produced by adipose tissue [32]. This overexpression leads to reduced macrophage activation and hence a decreased inflammatory response to antigen presentation. B-cell and T-cell responses are impaired as well. In the setting of H1N1 influenza, this has been linked to increased susceptibility to infections, prolonged time to clearing of infectious agents, decreased vaccine response, and the emergence of more virulent strains in obese persons [32].

Obesity induces a state of chronically elevated leptin and reduced adiponectin. This imbalance between proinflammatory and anti-inflammatory adipokines leads to a dysregulated immune response to a viral illness. Leptin also regulates B-cell maturation, development, and function [32]. Leptin resistance has been associated with disease severity in H1N1 influenza. Other pro-inflammatory cytokines produced by adipose tissue, including tumor necrosis factor alpha (TNF- $\alpha$ ), monocyte chemoattractant protein-1 (MCP-1), and interleukin-6 (IL-6), also increase the dysregulation [26, 32]. Physical activity is currently the best known nonpharmacological immunomodulatory intervention to improve disease outcomes in obese persons. Exercise decreases TNF $\alpha$, MCP-1, and IL-6, thus working to correct the underlying imbalance caused by obesity [32].

Obesity has also been correlated with lower numbers of CD8 T-cells and poorer quality of their immune response [32]. The impairment of CD8 T-cell response can diminish the immune system's ability to fight viral infection. Reductions in the antibody response and memory T-cell maturation may also negatively affect vaccine efficacy [32]. The phenomenon of a weakened immune response in obese patients has also been observed with the influenza vaccine [3••].

Adipose tissue serves as a main source of IL- 6 secretion, and IL-6 is responsible for activating multiple cytokine pathways, which may lead to a cascading effect or cytokine storm [3••]. A recent study by Ruan et al. [33] has demonstrated that IL-6 is an independent risk factor for severe COVID-19 infection.

Adipose tissue is important for maintaining normal immune, endocrine, and metabolic function in the human body [30]. It may act as a sponge that serves as a reservoir for viral shedding. It has been hypothesized that the hematogenous spread of viral particles or local spread from organs proximal to adipose deposits may place the virus in proximity to these processes and disrupt them. Such a relationship between adipose tissue and lung epithelium in $\mathrm{H} 5 \mathrm{~N} 1$ infection has been studied [30]. Increased adiposity has also been shown to be related to a $42 \%$ increase in the duration of shedding of the influenza virus compared to nonobese individuals [34].

The renin-angiotensin-aldosterone system plays a significant role in the pathophysiology of COVID-19 infection. COVID-19 enters cells by binding to the ACE2 receptors on the cells of the nasopharynx. ACE2 receptors are also expressed in the lungs, GI tract, and adipose tissue [35••]. As a result, the increased adipose tissue in obese patients could have an effect on viral load and outcomes from COVID-19 infection.

\section{Summary}

Hypertension and obesity play important roles in the COVID19 pandemic. As the numbers of individuals with obesity and hypertension rise across the globe, the collision of these three pandemics cannot be ignored. Understanding the mechanisms behind the associations between COVID-19 infection and hypertension and obesity is important in developing effective targeted therapies.

Furthermore, as vaccines become more accessible in the USA and abroad, the effects of RAAS and cytokine dysregulation on vaccine responses in the obese and hypertensive populations require further attention. Because it has not yet been determined how our current vaccines will respond to emerging variants of the COVID-19 virus, the focus on therapies targeting the ACE2 receptor and various cytokines should continue.

Since hypertension and obesity have been linked to increased risks of other viral infections, these more targeted therapies could make the healthcare community better prepared for epidemics and pandemics of the future.

The problems of obesity and hypertension are themselves of pandemic proportions. Thirty-eight million children under the age of 5 were overweight or obese in 2019, and over 240 million children and adolescents were overweight or obese in 2016 [23]. Of the estimated 1.13 billion people who have hypertension worldwide, fewer than $20 \%$ have controlled blood pressure. Physical inactivity, poor diet, and smoking are all well-established contributors to poorly controlled hypertension [36]. Interventions focusing on lifestyle modification and weight loss starting at a young age can be impactful not only in reducing the risk of comorbidities such as cardiovascular disease and chronic kidney disease, but also in decreasing the risk of severe complications from viral illnesses. 


\section{Declarations}

Conflict of Interest The authors declare no conflicts of interest relative to this manuscript

Human and Animal Rights and Informed Consent This article does not contain any studies with human or animal subjects performed by any of the authors

\section{References}

Papers of particular interest, published recently, have been highlighted as:

- Of importance

•- Of major importance

1. Hamming I, Cooper ME, Haagmans BL, Hooper NM, Korstanje R, Osterhaus ADME, et al. The emerging role of ACE2 in physiology and disease. J Pathol. 2007;212(1):1-11. https://doi.org/10.1002/ path. 2162 .

2. Fahme S, Bloomfield G, Peck R. Hypertension in HIV-infected adults: novel pathophysiologic mechanisms. Hypertension (Dallas, Tex 1979). 2018;72(1):44-55. https://doi.org/10.1161/ HYPERTENSIONAHA.118.10893.

3.• Aghili SMM, Ebrahimpur M, Arjmand B, et al. Obesity in COVID-19 era, implications for mechanisms, comorbidities, and prognosis: a review and meta-analysis. Int J Obes. 2005;2021:119. https://doi.org/10.1038/s41366-021-00776-8 This review article provides an in depth analysis of the dysregulation of many cytokines in the setting of obesity and how that affects immune response to viral illness.

4. Huang C, Wang Y, Li X, et al. Clinical features of patients infected with 2019 novel coronavirus in Wuhan, China. The Lancet (British edition). 2020;395(10223):497-506. https://doi.org/10.1016/ S0140-6736(20)30183-5.

5. Goyal P, Choi JJ, Pinheiro LC, et al. Clinical characteristics of COVID-19 in New York City. N Engl J Med. 2020;382(24): 2372-4. https://doi.org/10.1056/NEJMc2010419.

6.• Richardson S, Hirsch JS, Narasimhan M, et al. Presenting characteristics, comorbidities, and outcomes among 5700 patients hospitalized with COVID-19 in the New York City area. JAMA : the journal of the American Medical Association. 2020;323(20):2052 9. https://doi.org/10.1001/jama.2020.6775 This case series was one of the first and largest studies that observed a strong correlation between hypertension, obesity, and COVID-19 infection and severity.

7. Yoshida Y, Gillet SA, Brown MI, Zu Y, Wilson SM, Ahmed SJ, et al. Clinical characteristics and outcomes in women and men hospitalized for coronavirus disease 2019 in New Orleans. Biol Sex Differ. 2021;12(1):20. https://doi.org/10.1186/s13293-02100359-2.

8. Schönfeld D, Arias S, Bossio JC, Fernández H, Gozal D, PérezChada D. Clinical presentation and outcomes of the first patients with COVID-19 in argentina: results of 207079 cases from a national database. PLoS One. 2021;16(2):e0246793. https://doi.org/ 10.1371/journal.pone.0246793.

9. Ng WH, Tipih T, Makoah NA, et al. Comorbidities in SARS-CoV2 patients: a systematic review and meta-analysis. mBio. 2021;12:1. https://doi.org/10.1128/mBio.03647-20.

10. Grasselli G, Greco M, Zanella A, et al. Risk factors associated with mortality among patients with COVID-19 in intensive care units in
Lombardy, Italy. JAMA Intern Med. 2020;180(10):1345-55. https://doi.org/10.1001/jamainternmed.2020.3539.

11. Gupta S, Hayek SS, Wang W, et al. Factors associated with death in critically ill patients with coronavirus disease 2019 in the US. JAMA Intern Med. 2020;180(11):1436-46. https://doi.org/10. 1001/jamainternmed.2020.3596.

12. Cunningham JW, Vaduganathan M, Claggett BL, et al. Clinical outcomes in young US adults hospitalized with COVID-19. JAMA Intern Med. 2021;181(3):379-81. https://doi.org/10.1001/ jamainternmed.2020.5313.

13.• Wiese OJ, Allwood BW, Zemlin AE. COVID-19 and the reninangiotensin system (RAS): a spark that sets the forest alight? Med Hypotheses. 2020;144:110231. https://doi.org/10.1016/j.mehy. 2020.110231 This publication examines numerous mechanisms by which overstimulation of the RAAS pathway in COVID-19 infection causes the clinical manifestations of the illness.

14. Patel SK, Juno JA, Lee WS, et al. Plasma ACE2 activity is persistently elevated following SARS-CoV-2 infection: implications for COVID-19 pathogenesis and consequences. Eur Respir J. 2021;57: 1. https://doi.org/10.1183/13993003.03730-2020.

15. Vaduganathan M, Vardeny O, Michel T, McMurray JJV, Pfeffer MA, Solomon SD. Renin-angiotensin-aldosterone system inhibitors in patients with covid-19. N Engl J Med. 2020;382(17):16539. https://doi.org/10.1056/NEJMsr2005760.

16. Liu Y, Yang Y, Zhang C, Huang F, Wang F, Yuan J, et al. Clinical and biochemical indexes from 2019-nCoV infected patients linked to viral loads and lung injury. Sci China Life Sci. 2020;63(3):364 74. https://doi.org/10.1007/s11427-020-1643-8.

17. van de Veerdonk FL, Netea MG, Deuren M, et al. Kallikrein-kinin blockade in patients with COVID-19 to prevent acute respiratory distress syndrome. eLife. 2020;9. https://doi.org/10.7554/eLife. 57555.

18. Reynolds HR, Adhikari S, Pulgarin C, et al. Renin-angiotensinaldosterone system inhibitors and risk of covid-19. N Engl J Med. 2020;382(25):2441-8. https://doi.org/10.1056/NEJMoa2008975.

19. Ren L, Shandong Y, Wilson X, Overton J, Chiamvimonvat N, Phung T. Lack of association of antihypertensive drugs wtih the risk and severity of COVID-19: a meta-anaylsis. J Cardiol. 2021;77(5):482-91. https://doi.org/10.1016/j.jjcc.2020.10.015.

20. Biswas M, Kali MSK. Association of angiotensin-converting enzyme inhibitors and angiotensin-receptor blockers with risk of mortality, severity or SARS-CoV-2 test positivity in COVID-19 patients: meta-analysis. Sci Rep. 2021;11(1):5012. https://doi.org/10. 1038/s41598-021-84678-9.

21. Lanjanian H, Moazzam-Jazi M, Hedayati M, Akbarzadeh M, Guity K, Sedaghati-khayat B, et al. SARS-CoV-2 infection susceptibility influenced by ACE2 genetic polymorphisms: insights from tehran cardio-metabolic genetic study. Sci Rep. 2021;11(1):1529. https:// doi.org/10.1038/s41598-020-80325-x.

22. Wysocki J, Ye M, Hassler L, et al. A novel soluble ACE2 variant with prolonged duration of action neutralizes SARS-CoV-2 infection in human kidney organoids. J Am Soc Nephrol, 2021. https:// doi.org/10.1681/ASN.2020101537 This study demonstrates that a solube ACE2 receptor may serve as an effective treatment in neutralizing COVID 19 infection.

23. WHO obesity fact sheet. https://www.who.int/news-room/factsheets/detail/obesity-and-overweight Web site.

24. WHO health topics: Obesity. https://www.who.int/health-topics/ obesity\#tab=tab_1 Web site.

25. Popkin BM, Du S, Green WD, et al. Individuals with obesity and COVID-19: a global perspective on the epidemiology and biological relationships. Obes Rev. 2020;21(11):e13128. https://doi.org/ 10.1111/obr.13128.

26. Hendren N, de Lemos J, Ayers C, et al. Association of body mass index and age with morbidity and mortality in patients hospitalized 
with COVID-19: results from the american heart association COVID-19 cardiovascular disease registry. Circulation (New York, NY). 2020;143(2):135-44. https://doi.org/10.1161/ CIRCULATIONAHA.120.051936.

27. Anderson MR, Geleris J, Anderson DR, Zucker J, Nobel YR, Freedberg D, et al. Body mass index and risk for intubation or death in SARS-CoV-2 infection: a retrospective cohort study. Ann Intern Med. 2020;173(10):782-90. https://doi.org/10.7326/M20-3214.

28. Cai Q, Chen F, Wang T, Luo F, Liu X, Wu Q, et al. Obesity and COVID-19 severity in a designated hospital in Shenzhen, China. Diabetes Care. 2020;43(7):1392-8. https://doi.org/10.2337/dc200576 .

29. Kompaniyets L, Goodman AB, Belay B, et al. Body mass index and risk for COVID-19-related hospitalization, intensive care unit admission, invasive mechanical ventilation, and death-United States, March-December 2020. Morb Mortal Wkly Rep. 2021;70(10):35561 This large multicenter observational study adds further evidence of a link between obesity and risk of infection and severity of COVID-19.

30. Ryan PM, Caplice NM. Is adipose tissue a reservoir for viral spread, immune activation, and cytokine amplification in coronavirus disease 2019? Obesity (Silver Spring, Md). 2020;28(7):1191-4. https://doi.org/10.1002/oby.22843.

31. World health statistics 2020: Monitoring health for the SDGs, sustainable development goals. geneva: World Health Organization; 2020
32. Luzi L, Radaelli MG. Influenza and obesity: its odd relationship and the lessons for COVID-19 pandemic. Acta Diabetol. 2020;57(6):759-64. https://doi.org/10.1007/s00592-020-01522-8.

33. Ruan Q, Yang K, Wang W, Jiang L, Song J. Clinical predictors of mortality due to COVID-19 based on an analysis of data of 150 patients from Wuhan, China. Intensive Care Med. 2020;46(5):8468. https://doi.org/10.1007/s00134-020-05991-x.

34. Maier HE, Lopez R, Sanchez N, Ng S, Gresh L, Ojeda S, et al. Obesity increases the duration of influenza A virus shedding in adults. J Infect Dis. 2018;218(9):1378-82. https://doi.org/10.1093/ infdis/jiy370.

35.• Verdecchia P, Cavallini C, Spanevello A, Angeli F. COVID-19: ACE2centric infective disease? Hypertension (Dallas, Tex 1979). $2020 ; 76$ (2):294-9. https://doi.org/10.1161/ HYPERTENSIONAHA.120.15353 This review highlights the role of ACE2 receptors in COVID-19 infection and the imbalance in angiotensin II and angiotensin 1-7 that results with viral downregulation of these receptors.

36. WHO hypertension fact sheet. https://www.who.int/news-room/ fact-sheets/detail/hypertension Web site.

Publisher's Note Springer Nature remains neutral with regard to jurisdictional claims in published maps and institutional affiliations. 